\title{
Comparison of charged sheets and corrected 3-D Ewald calculations of long-range forces in slab geometry electrolyte systems with solvent molecules
}

\author{
Richard L. Rowley \\ rowley@byu.edu \\ Paul S. Crozier \\ Eckhard Spoht \\ Douglas Henderson
}

Follow this and additional works at: https://scholarsarchive.byu.edu/facpub

Part of the Chemical Engineering Commons

\section{Original Publication Citation}

P.S. Crozier, R.L. Rowley, E. Spohr, and D. Henderson, â€œComparison of charged sheets and corrected 3-D Ewald calculations of long-range forces in slab geometry electrolyte systems with solvent moleculesâ€, J. Chem. Phys. 112, 9253 (2)

\section{BYU ScholarsArchive Citation}

Rowley, Richard L.; Crozier, Paul S.; Spoht, Eckhard; and Henderson, Douglas, "Comparison of charged sheets and corrected 3-D Ewald calculations of long-range forces in slab geometry electrolyte systems with solvent molecules" (2000). Faculty Publications. 596.

https://scholarsarchive.byu.edu/facpub/596

This Peer-Reviewed Article is brought to you for free and open access by BYU ScholarsArchive. It has been accepted for inclusion in Faculty Publications by an authorized administrator of BYU ScholarsArchive. For more information, please contact ellen_amatangelo@byu.edu. 


\title{
Comparison of charged sheets and corrected 3D Ewald calculations of long-range forces in slab geometry electrolyte systems with solvent molecules
}

\author{
Paul S. Crozier ${ }^{\mathrm{a})}$ and Richard L. Rowley ${ }^{\mathrm{b})}$ \\ Department of Chemical Engineering, Brigham Young University, Provo, Utah 84602-4100 \\ Eckhard Spohr ${ }^{\mathrm{c})}$ \\ Department of Theoretical Chemistry, University of Ulm, Albert-Einstein-Allee 11, D-89069 Ulm, Germany \\ Douglas Henderson ${ }^{\text {d) }}$ \\ Department of Chemistry and Biochemistry, Brigham Young University, Provo, Utah 84602-5700
}

(Received 12 January 2000; accepted 7 March 2000)

\begin{abstract}
Two methods of calculating long-range intermolecular potentials are compared for an approximately $3 \mathrm{M}$ aqueous electrolyte solution confined between two charged surfaces. We investigate the ionic density profiles using the charged-sheets method and the corrected three-dimensional (3D) Ewald method at two different system sizes and also compare the Coulomb forces directly. The corrected 3D Ewald method is recommended for the calculation of long-range potentials in systems of this nature because it is less system size dependent than the charged-sheets method and the resultant forces are more consistent with periodic boundaries. However, the charged-sheets method for estimating long-range potentials in Coulombic systems may be useful for certain applications, and the corrected 3D Ewald method also shows some system size dependence. (C) 2000 American Institute of Physics. [S0021-9606(00)50421-8]
\end{abstract}

\section{INTRODUCTION}

Coulombic interactions between particles are long range in nature and must be properly calculated to correctly model electrolyte mixtures. ${ }^{1}$ There is much interest in the ability to model these interactions correctly since they are so prevalent and important in chemical and biological processes. Especially of interest is the modeling of interfacial regions, such as membrane surfaces, electrode surfaces, and vapor-liquid, liquid-liquid, or solid-liquid interfaces. These systems can be represented by slab geometry in Monte Carlo (MC) or molecular dynamics (MD) computer simulations where periodic boundary conditions are used in two of the three directions (i.e., the $x$ and $y$ directions) and a physical boundary prohibits passage of particles in the third direction (i.e., the $z$ direction). Such systems must necessarily use different methods for the long-range interactions acting beyond the primary cell than are used in a simulation with periodicity in all three directions.

Many methods have been proposed to approximate longrange interactions for systems with slab geometry, each varying in reliability and computational demand. ${ }^{2-11}$ The purpose of this paper is to explore two of these techniques: the 3D Ewald summation method, with the correction term, (EW3DC) as outlined by Yeh and Berkowitz, ${ }^{6}$ and the charged-sheets method (CS), which was originally proposed by Torrie and Valleau. ${ }^{7-9}$

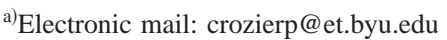

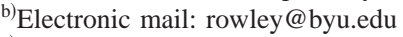

${ }^{c)}$ Electronic mail: eckhard.spohr@ @ chemie.uni-ulm.de

${ }^{\mathrm{d})}$ Electronic mail: doug@huey.byu.edu
}

Besides EW3DC and CS, other methods for approximating long-range interactions for systems of slab geometry have been developed. Pollack and Glosli ${ }^{11}$ indicate that the particle-particle particle-mesh method $\left(\mathrm{P}^{3} \mathrm{M}\right)$ and the fast multipole method (FMM) can both be adapted to slab geometry, and that both are generally more efficient than Ewald summation methods. They favor $\mathrm{P}^{3} \mathrm{M}$ as the most efficient and the easiest to implement of the methods. But for the purposes of this study, we confine our examination to the more common EW3DC and CS methods.

\section{LONG-RANGE POTENTIAL MODELS FOR SLAB GEOMETRY}

\section{A. The 3D Ewald summation with the correction term}

Perhaps the most widely used method for the approximation of long-range potentials acting beyond the primary cell is the Ewald sum method. ${ }^{1,12}$ This method was developed to model long-range interactions in a periodically repeating crystalline structure. Although the method introduces periodicity to what should be an amorphous system, it has been shown to model the structure and properties of real liquids reasonably when a primary cell of sufficient size is used (i.e., on the order of 100-10000 particles). ${ }^{1,5,13}$

Even though the Ewald sum method was originally developed for systems that are periodic in all three directions, Parry and others ${ }^{2-4}$ have developed the equations necessary for a slab geometry; i.e., a two-dimensional (2D) Ewald method (EW2D). The equation for the total Coulombic energy is given by 


$$
\begin{aligned}
U= & \frac{1}{4 \pi \varepsilon_{0}} \sum_{\mathbf{m}}^{\dagger} \sum_{i=1}^{N} \sum_{j=1}^{N} q_{i} q_{j} \frac{1}{2} \frac{\operatorname{erfc}\left(\alpha\left|\mathbf{r}_{i j}+\mathbf{m}\right|\right)}{\left|\mathbf{r}_{i j}+\mathbf{m}\right|}+\frac{1}{4 \pi \varepsilon_{0}} \sum_{i=1}^{N} \sum_{j=1}^{N} q_{i} q_{j} \sum_{h>0} \frac{\pi \cos \left(\mathbf{h} \cdot \mathbf{r}_{i j}\right)}{2 h A}\left\{\exp \left(h z_{i j}\right) \operatorname{erfc}\left(\alpha z_{i j}+\frac{h}{2 \alpha}\right)\right. \\
& \left.+\exp \left(-h z_{i j}\right) \operatorname{erfc}\left(-\alpha z_{i j}+\frac{h}{2 \alpha}\right)\right\}-\frac{1}{4 \pi \varepsilon_{0}} \sum_{i=1}^{N} \sum_{j=1}^{N} q_{i} q_{j} \frac{\pi}{A}\left[z_{i j} \operatorname{erf}\left(\alpha z_{i j}\right)+\frac{1}{\alpha \sqrt{\pi}} \exp \left[-\left(\alpha z_{i j}\right)^{2}\right]\right] \\
& -\frac{1}{4 \pi \varepsilon_{0}} \frac{\alpha}{\sqrt{\pi}} \sum_{i=1}^{N} q_{i}^{2}
\end{aligned}
$$

where $\mathbf{m}$ is the lattice vector $\left(m_{x} L_{x}, m_{y} L_{y}, 0\right)$ with cell integers $m_{x}$ and $m_{y}$ and simulation cell lengths of $L_{x}$ and $L_{y}$ in the $x$ and $y$ directions, $\mathbf{h}$ is the reciprocal lattice vector given by $\left[\left(2 \pi m_{x}^{\prime}\right) / L_{x},\left(2 \pi m_{y}^{\prime}\right) / L_{y}, 0\right]$ with integers $m_{x}^{\prime}$ and $m_{y}^{\prime}, A$ is the surface area of the cell at the slab surface, $L_{x} \times L_{y}$. The number of $\mathbf{m}$ and $\mathbf{h}$ vectors and $\alpha$ are adjustable parameters chosen for computational efficiency. The dagger indicates the omission of the $i=j$ term when $\mathbf{m}=0$; i.e., for direct self-interactions within the primary cell.

Spohr ${ }^{5}$ showed that the regular 3D Ewald method (EW3D) (with tin-foil boundary conditions) almost reproduces the EW2D results, provided that the box length in the $z$ direction is about five times larger than the box length in the lateral directions and that there is empty space of sufficient thickness in the basic periodic box. The EW3D method is computationally more efficient than EW2D since it does not involve a double sum in reciprocal space. Recently Yeh and Berkowitz ${ }^{6}$ have shown that the EW3D can be modified to produce results in even better agreement with EW2D. The potential energy equation for EW3D is

$$
\begin{aligned}
U= & \frac{1}{4 \pi \varepsilon_{0}} \sum_{\mathbf{n}}^{\dagger} \sum_{i=1}^{N} \sum_{j=1}^{N} q_{i} q_{j} \frac{1}{2} \frac{\operatorname{erfc}\left(\alpha\left|\mathbf{r}_{i j}+\mathbf{n}\right|\right.}{\left|\mathbf{r}_{i j}+\mathbf{n}\right|} \\
& +\frac{1}{\varepsilon_{0} V} \sum_{k>0} \frac{1}{k^{2}} \exp \left(\frac{-k^{2}}{4 \alpha^{2}}\right)\left\{\left|\sum_{i=1}^{N} q_{i} \cos \left(\mathbf{k} \cdot \mathbf{r}_{i}\right)\right|^{2}\right. \\
& \left.+\left|\sum_{i=1}^{N} q_{i} \sin \left(\mathbf{k} \cdot \mathbf{r}_{i}\right)\right|^{2}\right\}-\frac{1}{4 \pi \varepsilon_{0}} \frac{\alpha}{\sqrt{\pi}} \sum_{i=1}^{N} q_{i}^{2},
\end{aligned}
$$

where $V$ is the simulation cell volume given by $L_{x} \times L_{y}$ $\times L_{z}, \mathbf{n}$ is the lattice vector given by $\left(n_{x} L_{x}, n_{y} L_{y}, n_{z} L_{z}\right)$ with integers $n_{x}, n_{y}$, and $n_{z}, \mathbf{k}$ is the reciprocal lattice vector given by $\left[\left(2 \pi n_{x}^{\prime}\right) / L_{x},\left(2 \pi n_{y}^{\prime}\right) / L_{y},\left(2 \pi n_{z}^{\prime}\right) / L_{z}\right]$ with integers $n_{x}^{\prime}, n_{y}^{\prime}$, and $n_{z}^{\prime}$. Again, the dagger indicates the omission of the $i=j$ term when $\mathbf{n}=0$.

The EW3DC method consists of the modification of EW3D to account for the slab geometry, and necessitates the elongation of the primary cell in the $z$ direction (perpendicular to the slab), and the addition of a correction term to remove the forces due to the net dipole of the periodically repeating slabs. An empty space of three to five times the space of the fluid-occupied region is left between the repeating slabs to dampen out interslab interactions. With this large empty volume between the slabs, only the leading dipole term needs to be removed in order to isolate each of the individual slabs. This dipole correction term consists of an additional energy term

$$
U_{c}=\frac{1}{2 \varepsilon_{0} V} M_{z}^{2},
$$

which leads to the $z$-component force given by

$$
F_{i, z}=-\frac{q_{i}}{\varepsilon_{0} V} M_{z}
$$

where $M_{z}$ is the net dipole moment of the simulation cell in the $z$ direction.

\section{B. The charged-sheets method}

Torrie and Valleau ${ }^{7-9}$ suggest an alternative approach to calculating long-range Coulombic interactions for slabgeometry systems that is computationally inexpensive, easy to implement, and does not introduce any periodicity into the system. Only long-range forces in the $z$ direction are approximated, and long-range forces in the $x$ and $y$ directions are assumed to be negligible. We shall call this method the charged-sheets method (CS) because of its use of infinite sheets of charge to represent long-range interactions in the $z$ direction beyond the primary cell. The original CS by Torrie and Valleau ${ }^{8}$ uses uniformly spaced sheets with a surface charge that corresponds to a time- and space-averaged charge distribution of the central cell. Since information from previous configurations is used, calculations performed using the original CS are not rigorous Markov chains. Here, we use a more rigorous version of CS as given by Boda, Chan, and Henderson, ${ }^{10}$ where each particle has a corresponding charged sheet representing charges acting from outside of the primary cell. For a quadratic slab with $L_{x}=L_{y}=L$, the potential energy calculated using $\mathrm{CS}$ is given by

$$
\begin{aligned}
& U=\frac{1}{4 \pi \varepsilon_{0}}\left\{\sum_{i=1}^{N-1} \sum_{j=i+1}^{N} \frac{q_{i} q_{j}}{r_{i j}}\right. \\
& \left.\quad+\sum_{i=1}^{N} \sum_{j=1}^{N}[u(i, j, \infty)-u(i, j, L)]\right\}, \\
& u(i, j, W)=q_{i} \frac{q_{j}}{W^{2}}\left\{4 W \ln \left(\frac{0.5+r_{1}}{r_{2}}\right)-|z| E(z, W)\right\}, \\
& E(z, W)=2 \pi-4 \arctan \frac{4|z| r_{1}}{W},
\end{aligned}
$$




$$
r_{1}=\sqrt{0.5+\left(\frac{z}{W}\right)^{2}},
$$

and

$$
r_{2}=\sqrt{0.25+\left(\frac{z}{W}\right)^{2}} .
$$

In the above, $z=z_{i}-z_{j}$ and is the $z$ component of the distance between charges $i$ and $j$.

While CS is much less computationally demanding than EW3DC, it should be recognized that it was developed for systems without solvent molecules, and has usually been applied to larger simulation cells in which screening within the primary cell is more effective. With the exception of a study by Boda et al. ${ }^{14}$ this is the first study of which we are aware in which CS is used for a system containing discrete polar molecules. In the study of Boda et al. difficulties were encountered in dissolving the ions in the dipolar hard-sphere solvent. Those difficulties might be due, in part, to the use of CS.

\section{SIMULATION DETAILS}

In order to test the effectiveness of EW3DC and CS, simulations of idealized ions using both methods were performed on systems with a molecular solvent and compared. The SPC/E model for water was chosen as the solvent and the idealized ions were assigned Lennard-Jones (LJ) parameters identical to the LJ parameters for the oxygen-oxygen interaction in the SPC/E model, as well as a charge of $\pm e$, where $e$ is the magnitude of the electron charge.

All simulations were performed at $298.15 \mathrm{~K}$ with a constant ratio of 25 water molecules per anion-cation pair. A wall comprised of cations was held rigid at $z=0 \AA$ and a wall of anions was held rigid at $z=27.58 \AA$. Wall anions and cations were placed on a square lattice, at $1.5 \AA$ intervals, and each ion was given an $\mathrm{LJ} \sigma=1.5 \AA$, and an $\mathrm{LJ} \varepsilon / k$ $=50 \mathrm{~K}$. In addition, the walls were charged to $0.1 \mathrm{C} / \mathrm{m}^{2}$ by giving each wall ion a partial charge of $\pm 0.0140434 e$. Initial configurations were chosen as a random distribution of water molecules, anions, and cations.

Two system sizes were tested using both EW3DC and CS. The first, smaller system consisted of 600 water molecules, 24 cations, and 24 anions. The slab geometry was $25.5 \times 25.5 \times 27.58 \AA$, and 289 ions were fixed in place on a square lattice to represent each wall. The second, larger system consisted of 1200 water molecules, 48 cations, and 48 anions with a slab geometry given by $36.062 \times 36.062$ $\times 27.58 \AA$. In this case, the square lattice forming the walls was rotated $45 \mathrm{deg}$ so that the ions would line up on the periodic boundaries; 578 cations were fixed at $z=0 \AA$, and 578 anions were fixed at $z=27.58 \AA$ to form the walls. The length of the periodic box in the $z$ direction was set at $78 \AA$ in EW3DC calculations for both system sizes.

For each simulation size and method, at least ten simulations were performed, each from a unique random configuration of the mobile particles. Each simulation lasted ap-

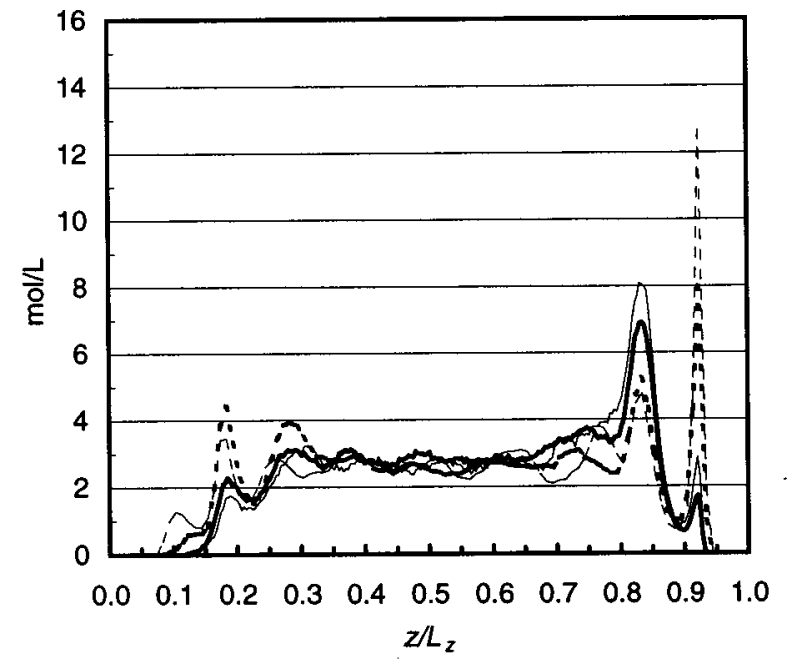

FIG. 1. Cation density profiles for the four cases. The traces are for the smaller system using EW3DC (-), larger system using EW3DC (-), smaller system using CS (---), and larger system using CS (---).

proximately 200 ps after approximately 20 ps of equilibration. Results shown below are averages over the simulation runs.

\section{RESULTS AND DISCUSSION}

\section{A. Simulation results}

Density profiles, as obtained using the two different sizes and two different long-range-force calculation methods are shown in Figs. 1 and 2 (cation distribution and anion distribution, respectively). As can be seen, the four cases are not equivalent. For of all, we note that the EW3DC runs do show considerably less contact adsorption than the CS runs.

The EW3DC results show a slight system size dependence, mostly in the first layer of cations and ions, while the CS results show a strong dependence on system size, especially in those first layers. Large peaks of anions and cations appear near the walls for the smaller simulation results ob-

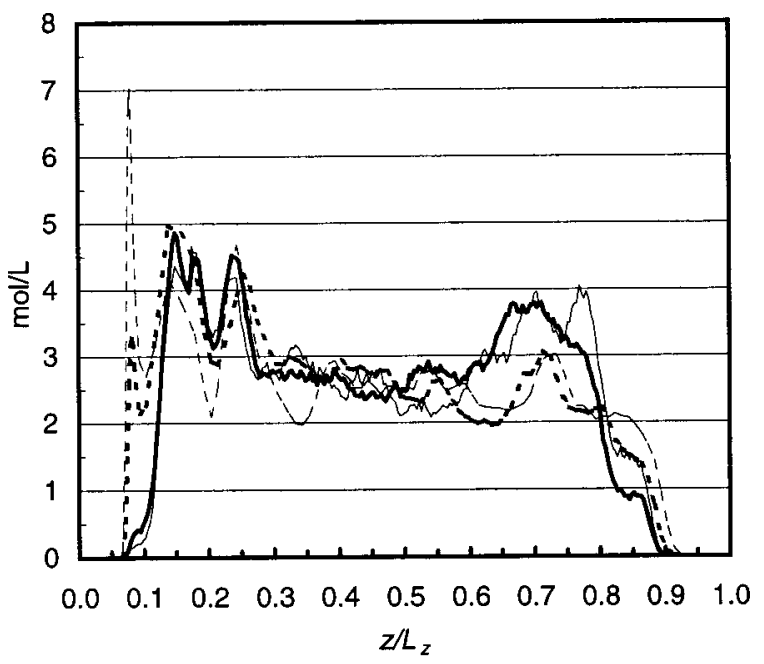

FIG. 2. Anion density profiles for the four cases. The traces are for the smaller system using EW3DC (-), larger system using EW3DC (-), smaller system using CS (---), and larger system using CS (---). 


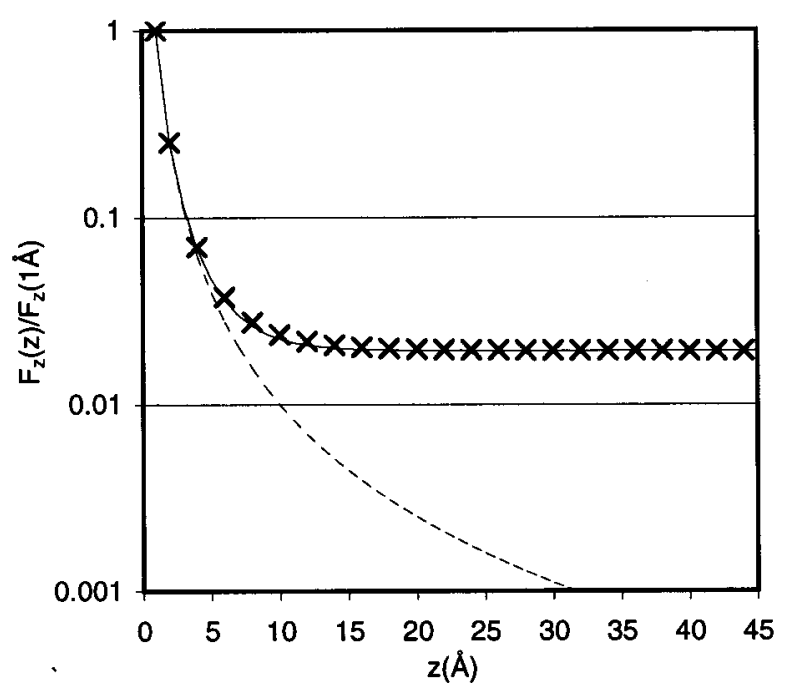

FIG. 3. Comparison of the force acting between two oppositely charged point charges in a two-dimensionally periodic system as calculated using EW2D or EW3DC (-), CS (×), and the bare Coulombic potential (------). The scale is chosen for comparison with Fig. 4 of Ref. 5 and Fig. 8 of 6.

tained using CS. As the system size is increased, the magnitudes of these peaks diminish and begin to resemble the EW3DC results.

\section{B. Forces between two particles}

To investigate the cause of the peak anomalies in the CS results near the wall surfaces, calculations of the total force on individual particles were made. This kind of direct comparison of forces has been utilized previously as a simple and effective way to elucidate the differences between various long-range-force calculation methods. ${ }^{5,6,15,16}$ Figure 3 shows a comparison between the forces acting in the $z$ direction between two oppositely charged particles as computed using a bare Coulomb potential, EW2D, EW3DC, and CS. Here, the same box geometry as Spohr ${ }^{5}$ and Yeh and Berkowitz ${ }^{6}$ was used for comparison purposes $\left(L=L_{x}=L_{y}=18 \AA\right.$ and $L_{z}=90 \AA$ ). The EW3DC and EW2D methods give excellent agreement in this case, while CS is almost, but not exactly the same. Figure 4 shows a comparison of forces parallel to the slab surface $\left(F_{y}\right)$ as calculated by EW3DC and CS, with the same box geometry as before. As can be seen, the forces as calculated by EW3DC necessarily go to zero at $L / 2$. This is because periodically repeating images of the particles are equidistant in both directions for this case, and therefore their forces perfectly counterbalance. On the other hand, CS produces a discontinuous jump in the calculated force as the minimum-image convention is applied at $L / 2$, because CS uses truncation in the $x$ and $y$ directions, rather than periodically repeating images. Both methods are only approximations to the infinitely extended nonperiodic slab. However, the discontinuous step in the lateral force of CS, the size of which depends on system size, is less satisfactory than the behavior of EW3DC, where the lateral force approaches zero at $L / 2$.

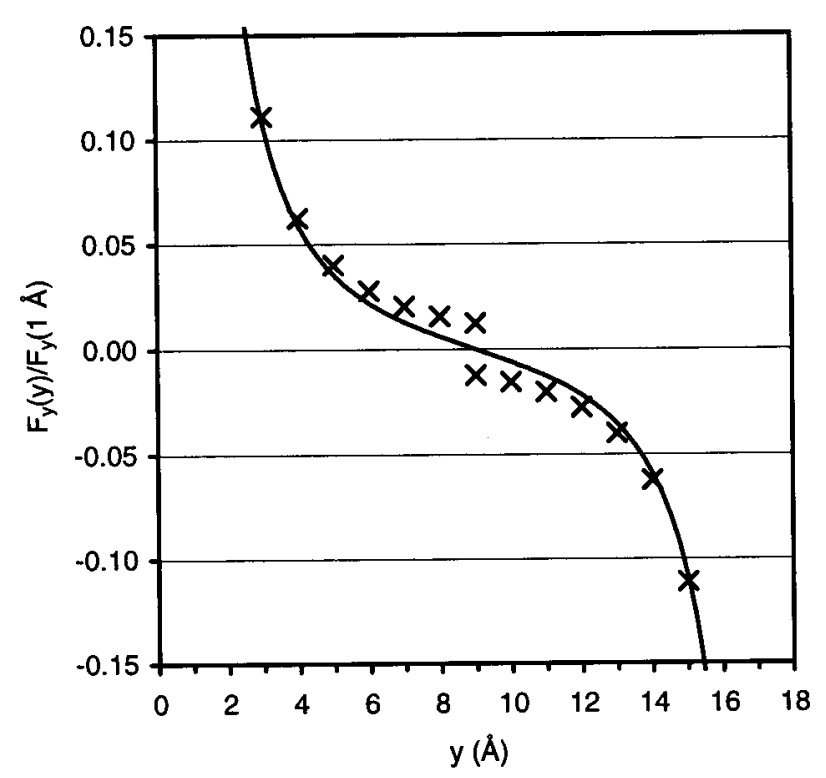

FIG. 4. Comparison of the $y$ component of force (parallel to the slab surface) acting between two oppositely charged point charges as calculated using EW3DC (-) and CS (X).

\section{Discussion}

The CS simulation results clearly show strong system size dependence. One simple explanation for this observation is that the forces in the $x$ and $y$ directions as calculated by CS are discontinuous at $0.5 \mathrm{~L}$. Since the comparison is between two systems, one containing 600 water molecules and one containing 1200 water molecules, of different $L$, the magnitude and location of the force discontinuity are not the same.

The discussion of the interaction forces between two point charges (Figs. 3 and 4) indicates that the large size dependence of CS is not so much due to the force in the $z$ direction as those in the $x$ and $y$ directions. Obviously, the larger the discontinuous jump in the lateral forces, the larger is the effect on the density profiles in Figs. 1 and 2. Although we did not explicitly study larger CS systems, we expect CS to yield results more similar to the EW3DC case as $L$ increases.

It is noteworthy that the forces in the $x$ and $y$ directions acting between an ion pair as calculated by EW3DC are also dependent upon the size of the simulation cell, but are not discontinuous at $0.5 \mathrm{~L}$. The artificial periodicity in the $x$ and $y$ directions causes this problem, and is endemic to the infinite slab geometry. The slight discrepancy between the 600water-molecule and 1200-water-molecule EW3DC density profiles is perhaps due to this periodicity. Torrie and Valleau ${ }^{8}$ likewise blame "spurious periodicity" for various unphysical results produced by Ewald-type lattice sums.

Periodicity in the $x$ and $y$ directions would not be eliminated by using EW2D instead of EW3DC. Yeh and Berkowitz $^{6}$ clearly demonstrate excellent agreement between EW2D and EW3DC in their work. Based on their conclusions, we can safely assume that EW2D calculations would show good agreement with our EW3DC calculations, and that system size effects that are observable in EW3DC calculations would also be evident in EW2D calculations. 
As pointed out earlier, previous applications of CS have been limited to primitive model calculations with no explicit solvent molecules at low ionic concentration (except for the study by Boda et $_{\text {al. }}{ }^{13}$ ). Neglect of explicit solvent molecules allows for a much larger simulation cell and screened Coulombic interactions. Interactions for such a system have much shorter-range effects, which explains why earlier studies involving CS produced satisfactory results which were shown to be reasonably independent of system size ${ }^{7-9} \mathrm{~A}$ simulation that includes solvent particles requires roughly two orders of magnitude more particles in order to simulate a system with the same number of ions as that of a system with solvent treated through the dielectric parameter. Thus, a much smaller simulation box must be used, given a fixed amount of available computation time. Smaller simulation boxes require a shorter truncation distance for the long-range interactions in the $x$ and $y$ directions. These truncations, in turn, can cause a distortion in the $z$ direction density profile as seen in Figs. 1 and 2. Correct calculation of the long-range forces in the $x$ and $y$ directions using CS for a system that includes solvent molecules would necessitate the use of a very large system size - on the order of 10000 solvent molecules or more-and becomes computationally prohibitive.

Performing molecular dynamics simulations using discontinuous forces between particle pairs as is necessitated by CS also causes an artificial heating that must be counteracted by a thermostat. ${ }^{17,18}$ In addition, the unnatural discontinuity requires the use of an unusually small time step to step through the discontinuity.

\section{v. CONCLUSION}

Due to the problems associated with using CS for the approximation of long-range forces in the $x$ and $y$ directions in electrolyte systems containing polar solvent molecules, its use is generally not recommended for systems of this nature. Although EW3DC is computationally more demanding than CS for a system of equal size, there is no abrupt minimumimage discontinuity in the $x$ and $y$ directions, and therefore there is a much smaller system size effect. Thus, we conclude that EW3DC is preferred over CS for use in simulations that contain solvent molecules. However, special cir- cumstances may warrant use of CS in order to save computer time (i.e., interactions between wall particles and bulk particles where $x$ and $y$ symmetry cancels any force discontinuities).

Even with EW3DC, caution must be used in system size selection in order to not introduce artifacts into simulation results. Figures 1 and 2 show that EW3DC does exhibit slight system size dependence. Due to the representation of an amorphous system by a periodic fluid as is required by EW3DC, we recommend the use of a simulation cell that is as large as feasible in order to mask the periodicity.

\section{ACKNOWLEDGMENTS}

The work of P.C., R.R., and D.H. was supported in part by the National Science Foundation (Grant No. CHE9813729) and by the donors of the Petroleum Research Fund, administered by the American Chemical Society (Grant No. ACS-PRF 31573-AC9). E.S. thanks D.H. for financial support and his hospitality.

${ }^{1}$ M. P. Allen and D. J. Tildesley, Computer Simulations of Liquids (Oxford University Press, New York, 1987).

${ }^{2}$ D. E. Parry, Surf. Sci. 49, 433 (1975); 54, 195 (1976).

${ }^{3}$ D. M. Heyes, M. Barber, and J. H. R. Clarke, J. Chem. Soc., Faraday Trans. 2 73, 1485 (1977).

${ }^{4}$ S. W. de Leeuw and J. W. Perram, Mol. Phys. 37, 1313 (1979).

${ }^{5}$ E. Spohr, J. Chem. Phys. 107, 6342 (1997).

${ }^{6}$ I.-C. Yeh and M. L. Berkowitz, J. Chem. Phys. 111, 3155 (1999).

${ }^{7}$ G. M. Torrie and J. P. Valleau, Chem. Phys. Lett. 65, 343 (1979).

${ }^{8}$ G. M. Torrie and J. P. Valleau, J. Chem. Phys. 73, 5807 (1980).

${ }^{9}$ G. M. Torrie, J. P. Valleau, and G. N. Patey, J. Chem. Phys. 76, 4615 (1982).

${ }^{10}$ D. Boda, K.-Y. Chan, and D. Henderson, J. Chem. Phys. 109, 7362 (1998).

${ }^{11}$ E. L. Pollock and J. Glosli, Comput. Phys. Commun. 95, 93 (1996).

${ }^{12}$ S. W. de Leeuw, J. W. Perram, and E. R. Smith, Proc. R. Soc. London, Ser. A 373, 27 (1980); 388, 177 (1983).

${ }^{13}$ D. M. Heyes, J. Chem. Soc., Faraday Trans. 90, 3039 (1994).

${ }^{14}$ D. Boda, D. Henderson, R. Rowley, and S. Sokolowski, J. Chem. Phys. 111, 9382 (1999).

${ }^{15}$ A. H. Widmann and D. B. Adolf, Comput. Phys. Commun. 107, 167 (1997).

${ }^{16}$ S. Y. Liem and J. H. R. Clarke, Mol. Phys. 92, 19 (1997).

${ }^{17}$ T. A. Andrea, W. C. Swope, and H. C. Anderson, J. Chem. Phys. 79, 4576 (1983).

${ }^{18}$ D. J. Adams, E. M. Adams, and G. J. Hills, Mol. Phys. 38, 387 (1979). 